\title{
Designing a Feature Vector for Statistical Texture Analysis of Brain Tumor
}

\author{
Kavitha Prithiviraj, S Prabakaran
}

\begin{abstract}
:
This paper presented a feature vector using a different statistical texture analysis of brain tumor from MRI image. The statistical feature texture is computed using GLCM (Gray Level Co-occurrence Matrices) of Brain Nodule structure. For this paper, the brain nodule segmented using strips method to implemented marker watershed image segmentation based on PSO (Particle Swarm Optimization) and Fuzzy C-means clustering (FCM). Furthermore, the four angles $0^{\circ}, 45^{\circ}, 90^{\circ}$ and $135^{\circ}$ are calculated the segmented brain image in GLCM. The four angular directions are calculated using texture features are correlation, energy, contrast and homogeneity. The texture analysis is performed a different types of images using past years. So the algorithm proposed statistical texture features are calculated for iterative image segmentation. These results show that MRI image can be implemented in a system of brain cancer detection.
\end{abstract}

Keywords: MRI image; texture features; GLCM

\section{INTRODUCTION}

In this research [1], proposed to detect microscopic brain image are used to pre-processed. The pre-processing image is extracted using texture feature in GLCM (Gray Level Co-Occurrence Matrix) method. Finally, the classification techniques using back propagation algorithm in neural network. Statistical textures features are extracted image based on features are contrast, correlation, energy, and homogeneity, whether the brain image are classified into cancerous and non-cancerous class using back propagation algorithm. In this proposed algorithm are classifying the input image with $95 \%$ of training set and $81.2 \%$ of testing set. This two various system are proved an implemented for brain cancer detection purpose.

The paper presented the construction of GLCM matrix using different angular directions and extracting their features. It's performed the direction of GLCM processing and the importance of SVM and PCA Further; we showed that the use of a PCA-SVM is far more optimal than the naïve classifier for classifying the brain cancer images [2].

The author is mainly focused to implement soft computing methods. In pre-processing, various filtering methods median, gabor filter and anisotropic filtering are compared and proved

P.Kavitha, Dept. of Information Technology, Bharath Institute of Higher Education and Research, Channai, India.

S.Prabakaran, Computer Science and Engineering, SRM University, Kattankulathur, India.
Anisotropic filter are more suitable for pre-processing. A Marker based Watershed algorithm is used to segment the brain nodule to extract the image. Finally, compared SVM and $\mathrm{KNN}$ classification result the KNN classifier to provide better accuracy value of the image and this proposed method was implemented by Shraddha G. Kulkarni, Sahebrao B. Bagal [3].

Ada, Ranjeet Kaur [4] was proposed, the group of images to sort according to the similarities and main objective of this paper using histogram equalization for pre-processing CT image. The pre-processing image is using feature extraction and to classify the better accuracy value using the method of Neural Network algorithm. Finally, the highest rate of True Positive (TP) and False Positive (FP) it give 96\% result was compared to other classification methods.

The proposed techniques are implemented by pre-processing to reduce noise reduction and smoothing of the original image quality was improved. In this article, the top-hat transform filter in morphological methods to extract the small unit of image pixel. The small extraction image computed by morphological opening and then the filter image is subtracted by original image. In segmentation, Otsu's method can perform cluster based thresholding and it convert into a binary image. It assumes two pixel classes compute the threshold value and separate two classes and the total variances and mean is calculating threshold value. A graythresh() method are used to perform Otsu thresholding. Watershed transformations to finding the watershed ridge line and catchment basins in an image. In classification, the support vector machine (SVM) to classify better accuracy $90 \%$ compared other classification method. The proposed method was implemented by Ayushi Shukla, Chinmay Parab and Pratik Patil [6].

\section{METHODOLOGY}

\section{A. Proposed Methods}

The earlier detection of brain tumor using Pre-processing techniques of top-hat transform, median and adaptive bilateral filter was compared both filtering methods and proved the adaptive bilateral filter is suitable method for MRI images. The proposed segmentation technique uses novel strip method and the image is split into number of strips 3, 4, 5 and 6. A marker watershed algorithm based on PSO (Particle Swarm Optimization) and Fuzzy C-mean Clustering (FCM) was proposed method. 
Firstly, the input image was reduced noise reduction and smoothing and the filter image is using strips method and then the image is segmented by marker watershed method. Secondly, the enhanced PSO technique was used to locate the better accurate value of the clustering centers of Fuzzy C-mean Clustering. Final stage, with the accurate value of centers and the enhanced target function and the small region of the segmented image was clustered by Fuzzy C-mean. The segmentation algorithm presented in this paper gives $95 \%$ of accuracy rate to detect brain tumor when strip count is 5 .

\section{B. GLCM Features}

The GLCM (Gray Level Co-occurrence Matrices) using various angular directions like $0^{\circ}, 45^{\circ}, 90^{\circ}$ and $135^{\circ}$. The GLCM considered the intensity resolution as 128 and 256. Totally the four texture features correlation, energy, contrast and homogeneity.

\section{Statistical Texture Analysis}

The sample images analyzed texture features correlation, energy, contrast and homogeneity.

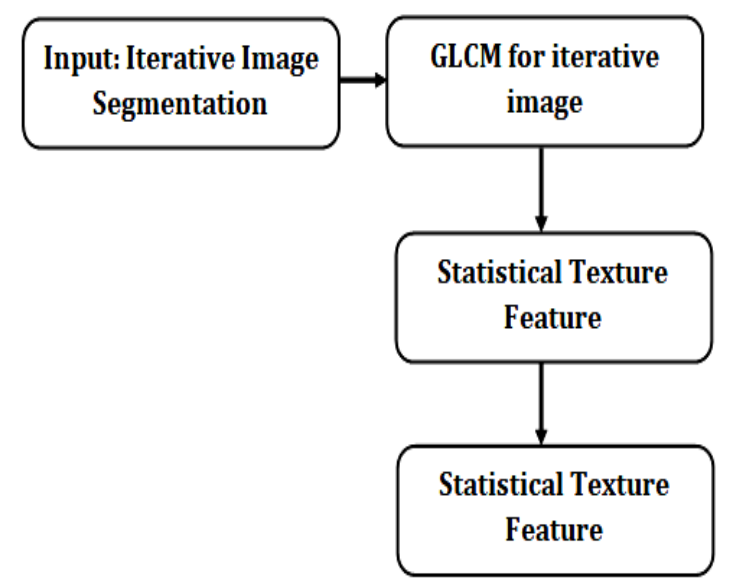

Figure1. Block Diagram of Proposed Method

Table1. Average of Strip str2 in Texture Feature

\begin{tabular}{|l|c|c|c|c|c|c|}
\hline $\begin{array}{l}\text { Angular } \\
\text { of Image } \\
\text { Intensity }\end{array}$ & \multicolumn{2}{|c|}{ offset } & Contrast & Correlation & Energy & Homogeneity \\
\hline $128,0^{\circ}$ & 0 & 1 & 61.2 & 0.96 & 0.019 & 0.9 \\
\hline $128,45^{\circ}$ & -1 & 1 & 138 & 0.9 & 0.017 & 0.62 \\
\hline $128,90^{\circ}$ & -1 & 0 & 95.1 & 0.94 & 0.015 & 0.73 \\
\hline $128,135^{\circ}$ & -1 & -1 & 94.3 & 0.94 & 0.014 & 0.68 \\
\hline $256,0^{\circ}$ & 0 & 1 & 289 & 0.96 & 0.019 & 0.78 \\
\hline $256,45^{\circ}$ & -1 & 1 & 569 & 0.9 & 0.017 & 0.54 \\
\hline $256,90^{\circ}$ & -1 & 0 & 379 & 0.94 & 0.015 & 0.68 \\
\hline $256,135^{\circ}$ & -1 & -1 & 374 & 0.94 & 0.014 & 0.63 \\
\hline
\end{tabular}

In an Image, it measures the local intensity value changes in contrast features. If the image intensity contains even the contrast value is zero. If the contrast value is high the image intensity value also increased. The correlation ranges from -1 to 1 and the correlation measures hoe the image pixel is correlated to other neighbor image pixels. The similarity of image pixel is measured using homogeneity. The maximum count of the pixel is similar intensity means the homogeneity is high. The minimum intensity changes are occurred in the textures.

The energy is also known as ASM (Angular Second Moment) it performs uniform intensity. The energy rate is 1 in the constant intensity image.

\section{RESULTS AND DISCUSSION}

The sample image was implemented in this proposed algorithm. The Table 1 shows the average of strip str2 in texture feature. The image intensity indicates 128 and 256 of gray-levels and the angular direction $0^{\circ}, 45^{\circ}, 90^{\circ}$ and $135^{\circ}$ using GLCM features. There are four GLCM features measure above are calculated using implemented methods and are analyzed. Contrast measure the high image intensity variations are present in the structure. In average contrast high intensity is 569 for angle $45^{\circ}$ for strip str2. The angle $0^{\circ}$ is minimal value of the contrast. So the figure 2 can observed that brain structure on some angle at strip str2.

The correlation measures the pixel are correlated to neighbour pixel the ranges from -1 to 1 . Correlation display the highest value of angle $0^{\circ}$ the vertical texture property is prominent. The homogeneity count is high in the angle of $0^{\circ}$ and it supports correlation that means those similar intensity pixels are presented in the vertical direction. The energy also measure high value in the vertical direction. The energy value is high in the angle of $0^{\circ}$ compared other angles. When the image intensity is reduced from 256 to 128 , the contrast value also reduced. But the value of energy, correlation and homogeneity has not changed even if image intensity count is reduced.

\section{CONCLUSION}

The spectrum of methods followed the designs of feature vector for brain nodule using statistical texture analysis. This paper mainly focused on statistical texture analysis using in brain cancer image using GLCM matrix. The Feature Analysis measure four texture features are energy, contrast, correlation and homogeneity with two image intensity 128 and 256. The image intensity is used to calculate four directions like $0^{\circ}, 45^{\circ}, 90^{\circ}$ and $135^{\circ}$ using GLCM features. It performs the minimum and maximum value of the different angle. In future, the statistical texture feature is very useful to analyzed structure of the image features.

\section{REFERENCES}

1. Pooja M, Veena Devi "A Hybrid Approach Based on Texture Feature Analysis in CT Images" International Journal of Advanced Research in Electrical, Electronics and Instrumentation Engineering Vol. 5, Issue 7, July 2016

2. Kusworo Adi, Catur Edi Widodo, Aris Puji Widodo "Detection Brain Cancer Using Gray Level Co-Occurrence Matrix (GLCM) and Back Propagation Neural Network Classification" Journal of Engineering Science and Technology Review 11 (2) (2018) 8- 12

3. Shraddha G. Kulkarni, Sahebrao B. Bagal "Brain Cancer Tumor Detection Using Image Processing and Soft Computing Techniques" International Journal of Science Technology and Management Volume 05 ISSN 2394-1537, May 2016

4. Ada, Rajneet Kaur, "Early Detection and Prediction of Brain Cancer Survival using Neural Network Classifier", IJAIEM volume 2, Issue 6 , June 2013 
5. Rashidul Hasan and Muntasir AI Kabir "Brain Cancer Detection and Classification based on Image Processing and Statistical Learning" DOI:10.13140 / RG.2.2.29499.46887

6. Ayushi Shukla, Chinmay Parab and Pratik Patil "Brain Cancer Detection using Image Processing Techniques" International Research Journal of Engineering and Technology (IRJET) Volume: 05 Issue: 04 | Apr-2018.

7. Rashmita Sehgal, Saurabh Gupta "Brain Cancer Detection Using Neural Networks" International Journal of Advanced Research in Computer Science and Software Engineering Volume 6, Issue 10, October 2016.

8. A. Asuntha1, A.Brindha1, S.Indirani1 "Brain cancer detection using SVM algorithm and optimization techniques" Journal of Chemical and Pharmaceutical Sciences Volume 9 Issue 4 ISSN: 0974-2115.

9. Bariqi Abdillah, Alhadi Bustamam, and Devvi Sarwinda "Image processing based detection of brain cancer on CT scan images" The Asian Mathematical Conference 2016 (AMC 2016) IOP Conf. Series: Journal of Physics: Conf. Series 893 (2017) 012063.

10. Raviprakash S. Shriwas, Akshay D. Dikondawar "Brain Cancer Detection And Prediction By Using Neural Network" IPASJ International Journal of Electronics \& Communication(IIJEC) volume 3, Issue 1, January 2015

11. Preeti Panwar, Girdhar Gopal, Rakesh Kumar "Image Segmentation using K-means clustering and Thresholding” International Research Journal of Engineering and Technology (IRJET) Volume: 03 Issue: 05 | May-2016

12. Preeti Panwar, Girdhar Gopal, Rakesh Kumar "Image Segmentation using K-means clustering and Thresholding" International Research Journal of Engineering and Technology (IRJET) Volume: 03 Issue: 05 | May-2016

13. S. Panda, "Color Image Segmentation Using K-means Clustering and Thresholding Technique,” IJESC, March 2015.

\section{Authors ProfiLe}

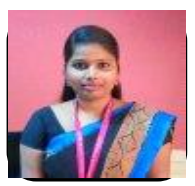

P Kavitha, Assistant Professor, Department of Information Technology, Bharath Institute of Higher Education and Research.

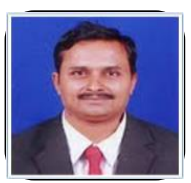

S.Prabakaran, Professor, Department of Computer Science and Engineering, SRM University 